\title{
Meta photonic crystal paper devices
}

\author{
GUO MaoZe ${ }^{1}$, WANG YuQiu ${ }^{2}$, HE BingFang ${ }^{1,2^{*}}$ \& GAO BingBing ${ }^{1 *}$ \\ ${ }^{1}$ School of Pharmaceutical Sciences, Nanjing Tech University, Nanjing 211816, China; \\ ${ }^{2}$ College of Biotechnology and Pharmaceutical Engineering, Nanjing Tech University, Nanjing 211816, China
}

Received May 7, 2020; accepted July 10, 2020; published online October 12, 2020

Citation: Guo M Z, Wang Y Q, He B F, et al. Meta photonic crystal paper devices. Sci China Tech Sci, 2020, 63: 2464-2466, https://doi.org/10.1007/s11431020-1690-y

With its unique properties of thin, porous, flexible and affordable, paper has been widely used as a multifunctional material in daily life since its invention [1]. Commonly, paper is the most popular carrier for information transmission such as writing and printing. However, since the applications of paper has been broadened continuously, it has also shown great prospect in the field of biochemical analysis. Paper-based analytical devices are easy to use, low cost, portable and disposable, thus playing an important role in the field of rapid detection [2].

With the development of paper-based materials, the identification of paper is no longer strict: membranes with flexible or porous structures could also be defined as "paper" [3]. Among various paper-based devices, photonic crystal (PC) paper has gained more attention. Photonic crystals (PCs) are regular optical structures due to the periodically arranged media of various refractive indices, occurring in nature in the form of structural coloration such as wings of some butterflies and skin of chameleons [4]. PC paper is a kind of flexible membrane devices which combined with PC structures. PC structure has photonic bandgap characteristics, which could allow light of a specific wavelength go through the structure while reflect the light of other wavelength. Therefore, compared with traditional cellulose paper, PC paper possesses unique optical properties. Currently, considerable PC paper devices are widely used in the field of biochemical analysis [5-9]. However, these devices suffer the monotonous function and poor adherence. It is highly

\footnotetext{
*Corresponding authors (email: bingfanghe@njtech.edu.cn; gaobb@njtech.edu.cn)
}

desired to design a multifunctional and super adhesive PC paper device.

Recently, inspired by tree frog's toe pads and gecko feet microstructures, Gu's group [10] designed a PC paper device with micropillar array structure by simply self-assembled elastic P(MMA-BA) copolymer nanoparticles on a micropatterned polydimethylsiloxane (PDMS) substrates for multifunctional applications (Figure 1(a)). Thanks to the micropillar array structure, the device achieved features as super adherence and spontaneous liquid transfer. The ordered microbionic arrays exhibit the great ability of interacting extensively with almost any surface, thereby enabling the device to adhere to skin surfaces stably. For mechanism, the device also showed significant mechanical property. In nanoindentation experiments and stretch-and-recover tests, the device could be completely restored under dry or wet condition after removing the strain, indicating that the device possessed excellent reversible mechanical property. In addition, Elastic polymer nanoparticles were self-assembled during the drying process and the transparent device can show vivid structure color after liquid was introduced, thus enhanced fluorescence (Figure 1(b)). This novel design successfully addresses the poor adherence of conventional PC paper, achieving multifunctional applications on PC paper.

It is found that when liquid was introduced on the PC paper, the highly ordered micropillar structures can manipulate the fluids, thereby microfluidics applications such as biochemical analysis could be achieved. To demonstrate the applicability of the PC paper in biological analysis, two 
(a)

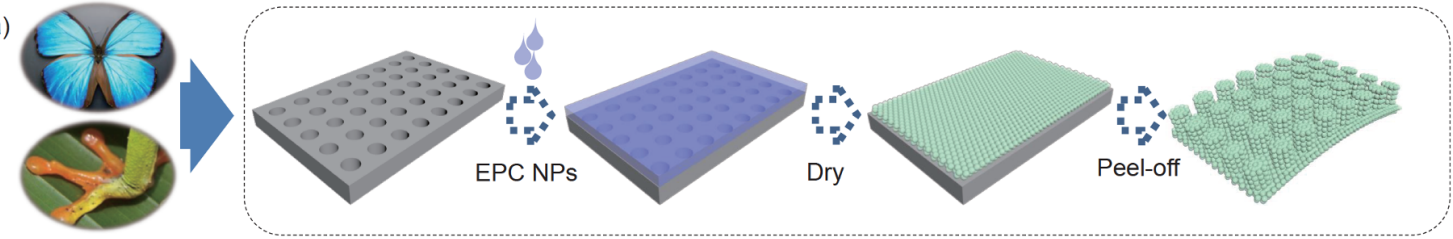

(b)

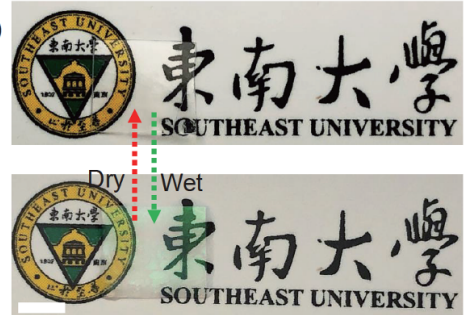

(c)

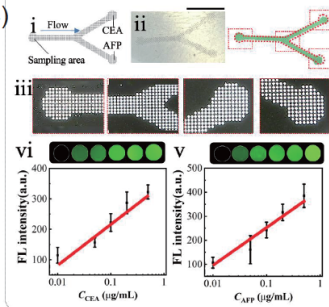

(d)

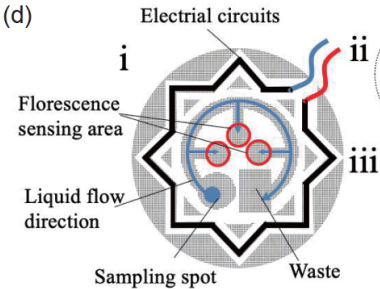

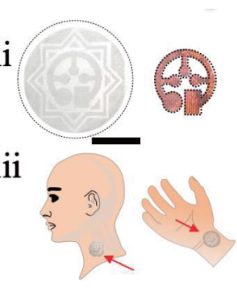
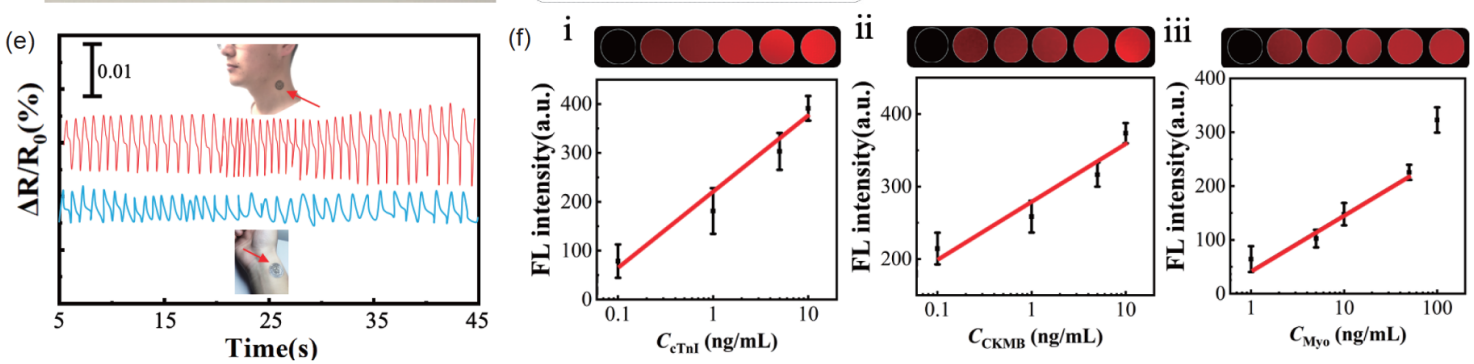

Figure 1 (Color online) Flexible photonic crystal paper for multifunctional applications. (a) Schematic diagram of the PC paper; (b) structure color transformation of the PC paper; (c) images of a Y shaped channels on the PC paper for biochemical analysis and the results; (d) schematic images of the skin chip; (e) relative resistance changes for the skin chips attached to volunteer's wrist and carotid artery for pulse sensing; (f) detecting of cardiac markers using the skin chips and the results. Reproduced with permission from ref. [10], Copyright 2019 Wiley-VCH Verlag GmbH \& Co. KGaA.

biomarkers (carcinoembryonic antigen (CEA) and alpha fetoprotein (AFP)) related to cancer diagnosis were selected for fluorescence detection (Figure 1(c)). The results show that both CEA and AFP concentrations have linear relationship with the fluorescence intensity, indicating the PC paper has great prospect in biochemical analysis.

In addition to biochemical analysis, the PC paper has potential application for skin chips as well. Due to the micropillar structure, the PC paper can stick stably to skin, sensing health situations of wearers. To demonstrate it, a PC paper skin chip with microfluidic channels and electronic circuits was fabricated by researchers (Figure 1(d)). The devices then were attaching to volunteers' wrist or carotid artery for cardiac markers and pulse sensing. The relative resistance change of the chip showed the pulse frequency of Volunteer 1 on wrist was $72 \mathrm{BMP}$, while for Volunteer 2 on carotid artery the number was $61 \mathrm{BMP}$, indicating that the sensors were capable of pulse sensing on different body parts (Figure 1 (e)). Furthermore, three biomarkers (cTnl, CKMB and MYO) for early diagnosis of acute myocardial infarction (AMI) were detected by the devices. The results show that the fluorescence intensity has a linear relationship with concentrations of cTnl, CKMB and MYO, which proved that the devices were capable of sensing cardiovascular markers (Figure 1(f)).

In general, this study provides a novel, nature-inspired, free-standing PC paper with microstructures. Due to the highly ordered microstructures (micro column) and nanostructures (PC structures), functions such as spontaneous liquid transferring, fluorescence enhancement and intimate skin contact can be achieved. The PC paper shows a great tolerance to body fluids conditions and dramatic biocompatibility. This research not only demonstrates the enormous potential applications of PC paper in various fields, but also represents a giant leap forward to the development of smart flexible PC paper devices. It can be anticipated that highly integrated and multifunctional PC paper devices can be created and applied in various fields such as point-of-care testing, soft robotics, organ on chips and healthcare.

This work was supported by the National Key Research and Development Program of China (Grant No. 2019YFA09005200), the National Natural Science Foundation of China (Grant No. 81673321), the Jiangsu Synergetic Innovation Center for Advanced Bio-Manufacture (Grant No. XTC1812) and the Special Funds for the Introduction of Talents of Nanjing Tech University (Grant No. 39828122).

1 Olson D R. The World on Paper: The Conceptual and Cognitive Implications of Writing and Reading. Cambridge: Cambridge University Press, 1996

2 Hu J, Wang S Q, Wang L, et al. Advances in paper-based point-of-care diagnostics. Biosens Bioelectron, 2014, 54: 585-597

3 Gao B, Liu H, Gu Z. Flourishing smart flexible membranes beyond paper. Anal Chem, 2019, 91: 4224-4234

4 Chen S, Guo L, Ji M, et al. Photonic crystal enhanced laser desorption and ionization substrate for detection of stress biomarkers under atmospheric pressure. J Mater Chem B, 2019, 7: 908-914 
5 Chi J, Shao C, Du X, et al. Generating microdroplet array on photonic pseudo-paper for absolute quantification of nucleic acids. ACS Appl Mater Interfaces, 2018, 10: 39144-39150

6 Chi J, Ma B, Dong X, et al. A bio-inspired photonic nitrocellulose array for ultrasensitive assays of single nucleic acids. Analyst, 2018, 143: 4559-4565

7 Zhang D, Gao B, Zhao C, et al. Visualized quantitation of trace nucleic acids based on the coffee-ring effect on colloid-crystal substrates.
Langmuir, 2019, 35: 248-253

8 Liao J, Zhu C, Gao B, et al. Multiresponsive elastic colloidal crystals for reversible structural color patterns. Adv Funct Mater, 2019, 29: 1902954

9 Gao B, Yang Y, Liao J, et al. Bioinspired multistructured paper microfluidics for POCT. Lab Chip, 2019, 19: 3602-3608

10 Gao B, Liao J, Guo M, et al. Biomimetic meta-structured electromicrofluidics. Adv Funct Mater, 2019, 29: 1906745 